\title{
CLUB-FORMS OF MYCOBACTERIUM LEPRAE
}

\author{
M. J. WISE \\ Hospital for Tropical Diseases, London.
}

Besides the well known granular forms of $M$. leprae, one may frequently observe larger bodies about twice the thickness of the bacillus. These have been recognised for many years. They were described in unstained preparations by SHOLTZ and KLINGMüLLER (1900). They are acid-fast and metachromatic with polychrome methylene blue (BABES quoted by KLINGMüLler 1900). Although described as 'spore-like' they are not the structures which NeISSER at one time took to be spores (these were the vacuolar spaces between the granules according to KLINGMüLLER) nor are they related to the Much's granules of mycobacteria which are not acid-fast and are probably artifacts (TOPLEY and WILSON, 1955). It is recognised however that club-like swellings similar to those of the diptheria bacillus occur not uncommonly in cultures of mycobacteria (TOPLEY and WILSON). Hereafter they are referred to as 'clubs'.

More recently a number of writers have referred to 'condensations' or 'metachromatic granules' without always making it clear whether these are the club-like swellings. DeNNEY however, illustrated them in 1934. De Souza Araujo (1959) described them clearly and found them to be visible under phase-contrast microscopy. He postulated that they might be concerned with regeneration of leprosy bacilli. It is surprising therefore that electron microscopy studies frequently omit any reference to 'clubs'; there are exceptions: Brieger and Glauert (1956). OKardo (1958) demonstrated them in electron-micrograms of $M$. lepraemurium.

This paper describes further observations on such club-forms.

\section{Material and Methods}

Skin smears and biopsies were received as a routine from all patients at the Jordan Hospital, Redhill. Smears from Sungei Buloh were also included. Biopsies before treatment were followed up at six monthly intervals during treatment and smears were examined at six weekly intervals for the first six months, and thereafter at intervals of six months. Details of classification and length of treatment were made available. Classification was of the system described by RIDLEY and JopLING (1962). The five groups are here referred to as tuberculoid, near-tuberculoid, borderline, nearleproma and leproma.

Although sections stained by the Fite-Faraco procedure following fixation in the F.M.A. fixative of Lowy (1956) showed the clubs clearly, they were not suitable for study because bacilli were usually in clumps. Skin smears or impression smears of biopsies were preferred. 
1. Smears were heat-fixed and stained by the Ziehl-Neelsen method. The granularity index was determined as described by RIDLEY (1960). (By this method an index of 0 represents all solid bacilli and $\mathrm{I} 0$ all granular forms.) To indicate the number of clubs an empirical scale of,,++++++ was employed.

2. Contact impressions of freshly excised skin biopsies were used for phase-contrast and for multiple histochemical studies. The correlation of stained structures with clubs was checked by phasecontrast or by restaining with Ziehl-Neelsen. The following stains were used:

(a) Vital staining using fresh contact impressions taken on slides prepared with a $1 / 15$ mixture of $0.4 \%$ Janus green and $0.25 \%$ neutral red and dried in a dust-free atmosphere.

(b) Fat stains, exposure to Osmium tetroxide and Baker's method for phospholipids.

(c) Gomori's Alkaline Phosphatase, with controls for specificity.

(d) Standard Feulgen.

(e) Periodic acid-Schiff (P.A.S.).

( $f$ ) Metachromatic methods and Mayer's mucihaematin.

(g) The Millon reaction for amino acids.

(h) Spore stains.

(i) Clubs were also examined by fluorescent and polariscopic procedures.

3. Solubility tests in common fat solvents were carried out.

4. Club forms of $M$. tuberculosis in actively growing young cultures were compared with those of $M$. leprae by phase-contrast microscopy, solubility and staining by P.A.S. and metachromatic methods.

\section{Results}

In all cases clubs were not numerous in untreated cases of lepromatous leprosy and in active lesions of relapsing leprosy. Untreated borderline patients with fairly large numbers of bacilli showed few clubs with a marked numerical variation between the lesions from which smears were made. In the near-tuberculoid cases with bacilli solid but scanty there were no clubs.

The number of clubs appeared to decline in proportion to an increase in granularity index with the improvement of the patient under treatment. On average, in lepromatous patients there was a general fall from +++ to ++ after the first six months of treatment. Thereafter numbers varied greatly at different sites. Where granularity was in the region of 9-10 in cases on treatment for several years, clubs were negligible. In borderline patients response to treatment produced a fall from +++ to - in the first three to six months, and afterwards they were not seen at all.

Clubs were strongly acid-fast in the routine Ziehl-Neelsen preparations. Once stained they held the dye tenaciously, much more 
so than the bacillary body itself. Usually they occurred in the solid uniformly stained bacilli. With treatment these bacilli were pale though still solid.

Clubs were located mainly towards the poles of the organism usually only one and sometimes two, one at either end. Less frequently the bacillus was fragmented and the club was seen in the centre. Never were there more than three in the same organism and this occurred only very rarely. Sometimes under phase-contrast microscopy 'the pedunculated comet-like' forms of De SouzA Araujo (1959) were seen, the bacillary bodies appearing as very short rods apparently emanating from the clubs.

Fluorescent staining presented the clubs as condensations rather than 'swellings'. These were isotropic and stained well with ordinary fat stains. No phospholipids were demonstrated but the clubs were strongly osmiophilic. They were P.A.S. positive and showed a $\gamma$-metachromasia with polychrome methylene blue and toluidine blue. Small granules within the bacillus reacted positively to the Feulgen technique, but these did not correlate with the clubs which were Feulgen negative. The bacillary body stained diffusely with Gomori's Alkaline Phosphatase and the clubs reacted strongly to it. They did not stain with spore stains or the Millon reaction. Vital staining was inconclusive.

Following extraction with xylol or chloroform clubs were not stainable. Staining was partially restored by subsequent soaking in oil and staining by Fite-Faraco's method. Clubs were insoluble in alcohol and acetone and resisted acids. After alkali their demonstration was difficult.

Club-forms of $M$. tuberculosis were seen only in actively growing young cultures. They occurred much less frequently than those of $M$. leprae. They were P.A.S. positive with a $\gamma$-metachromasia and insoluble in acetone. Because they were scanty they were difficult to study. But as far as could be demonstrated they were the same type of structure as the leprosy clubs.

In an attempt to explain why clubs are often unmentioned in studies of $M$. leprae by electron-micrographs, Dr. R. J. W. ReEs and Dr. R. C. VALENTINE undertook to examine a preparation containing clubs by electron microscopy. Material was provided from a lepromatous patient treated for $3 \frac{1}{2}$ years; the granularity index was 9 and there were no clubs in any of the smears save one, taken from a lesion obviously in a state of activity. This site was chosen for biopsy. The granularity index here was placed at 1 and the number of clubs +++ . Contact impressions were made from the excised tissue before homogenising in preparation for electron microscopy, and afterwards smears were prepared from the homogenate. Both smears were stained by Ziehl-Neelsen. Clubs were plentiful in the preparation before homogenization; none at all were seen after. 
The presumption was that the clubs had been destroyed during the process of homogenization of the tissue.

\section{Discussion}

The fact that clubs can be seen in unfixed, unstained, wet films is proof that they are not artifacts of drying, heat fixation or staining. They are strongly acid-fast and must therefore contain the constituent of mycobacteria which combines with carbol-fuchsin (mycolic acid and its derivatives). Their reaction to fat stains and solvents and to P.A.S. indicates that they contain a lipopolysaccharide complex and their reaction to Gomori's Alkaline Phosphatase shows the presence of this enzyme. In their absence of R.N.A. clubs differ from the volutin granules seen in some plant and animal cells. From the ease with which they are removed or are no longer demonstrable one assumes that they occupy a superficial position in relation to the organism. J. H. HANKS (1962) in studying the capsular components of leprosy bacilli, came to the conclusion that there was a double membrane in the organism, the outer one being easily destructible. Possibly the clubs lie between the two cell walls. After homogenization bacilli still retained their acid-fastnessa property dependent on an intact cell wall. One of the cell membranes must therefore have remained whole during the process. To preserve clubs for study by electron microscopy Dr. REES suggests that ultra thin sections rather than homogenates should be used.

The significance of clubs in $M$. leprae has not been fully elucidated. The questions are whether they have the same significance as other granular forms of this bacillus, and if not whether they are concerned with its viability or with a particular phase of its life cycle. It is clear from the results that clubs are not to be considered as granules; in their incidence and properties they are quite different. The fact that clubs decrease fairly quickly with treatment indicates that they are not the result of chemotherapy or of involution and death through this means. The finding that clubs are most numerous among solid-staining forms of bacilli, and in lepromatous rather than borderline types of leprosy, suggests that they are associated with growth, or possibly with the earliest phase of natural involution. It may be significant that clubs were prominent in the earliest phase of relapse when treatment was interrupted.

\section{Summary}

Club forms of Mycobacterium leprae have been studied in relation to the state of the bacillus and to treatment, by solubility tests and special stains, and by phase-contrast and electron microscopy.

Clubs were found to contain an acid-fast lipo-polysaccharide complex which was removed during the process of tissue homogenization prior to electron microscopy.

Clubs are not related to other acid-fast granules or to volutin. 
They appear to be associated with the growth phase of the bacillus but their significance remains uncertain.

\section{Acknowledgements}

I am very grateful to Dr. D. S. Ridley for his encouragement, and many useful suggestions and criticisms of this work; to Dr. R. J. W. Rees and Dr. R. C. Valentine, National Institute for Medical Research, London, for their help with the electron microscopy; also to Dr. W. H. JoplinG, Jordan Hospital, and Dr. M. F .R. WATERS, Sungei Buloh Leprosarium, Malaya, for their co-operation in preparing the smears and biopsies.

\section{References}

Brieger, E. M. and Glauert, A. M., Tubercle. London 1956. 3. 195.

De Souza-Araujo, H. C. Lep. Review 1959. 30. 80.

DENNEY, O. E., Int: J. Lep. 1934. 2, 275.

HANKS, J. H. Int. J. Lep. 1961, 29, 74.

KLINGmüller V. Die Lepra. Handbuch der Haut, und Geschlects. Krankheiten, vol. 10. Berlin: J. Springer, 1930, pp. 79-80.

Lowy, L. J. Med. Lab. Techn. 1956, 13, 558.

OKARDO, S. Research Activities of National Institute for Lep. Research. (special issue of Leprosy in Japan, 1958) p. 98.

RIDLEY, D. S. Int.J. Lep. 1960, 38, 3.

Ridley, D. S. and JopilnG, W. H. Lep. Review 1962, 33, 119.

Sholtz, W. and KlingmülleR, V. Lepra. (Leipzig) 1900.1.93.

TOPLEY and WILSON's Principles of Bacteriology and Immunity. 4th Edit. London, Arnold. 1955. pp. 491-495. 